\title{
Tumor-associated MUC5AC stimulates in vivo tumorigenicity of human pancreatic cancer
}

\author{
HIROTAKA HOSHI $^{1,3}$, TETSUJI SAWADA ${ }^{2}$, MOTOYUKI UCHIDA $^{1}$, HIKARU SAITO $^{1}$, HIROKO IIJIMA ${ }^{1}$, \\ MIKAKO TODA-AGETSUMA ${ }^{1}$, TSUTOMU WADA ${ }^{1}$, SADAAKI YAMAZOE ${ }^{2}$, HIROAKI TANAKA $^{2}$, \\ KENJIRO KIMURA ${ }^{2}$, ANNA KAKEHASHI $^{3}$, MIN WEI $^{3}$, KOSEI HIRAKAWA ${ }^{2}$ and HIDEKI WANIBUCHI ${ }^{3}$ \\ ${ }^{1}$ Biomedical Research Laboratories, Kureha Corporation, 3-26-2 Hyakunin-cho, Shinjuku-ku, \\ Tokyo 169-8503; Departments of ${ }^{2}$ Surgical Oncology and ${ }^{3}$ Pathology, Osaka City University \\ Graduate School of Medicine, 1-4-3 Asahi-machi, Abeno-ku, Osaka 545-8585, Japan
}

Received September 30, 2010; Accepted November 8, 2010

DOI: $10.3892 /$ ijo.2011.911

\begin{abstract}
MUC5AC, a high molecular weight glycoprotein, is overexpressed in the ductal region of human pancreatic cancer but is not detectable in the normal pancreas, suggesting its association with disease development. In the present study, we investigated the in vitro and in vivo effects of MUC5AC knockdown by short interfering RNA (siRNA) in the MUC5AC-overexpressing SW1990 and BxPC3 human pancreatic cancer cell lines in order to clarify its function. Significant decreases in the expression levels of MUC5AC mRNA and protein were observed in SW1990 and BxPC3 cells that had been stably transfected with a MUC5AC siRNA expression vector (SW1990/si-MUC5AC and BxPC3/siMUC5AC cells) compared to those in cells transfected with an si-mock vector (SW1990/si-mock and BxPC3/si-mock cells). In in vitro studies, neither type of MUC5AC-knockdown cell showed any difference in cell survival, proliferation, or morphology from the si-mock cells or parental cells. However, in vivo xenograft studies demonstrated that MUC5AC knockdown significantly reduced the tumorigenicity and suppressed the tumor growth of si-MUC5AC cells compared to those of the si-mock cells. Immunohistochemical analysis revealed that $\mathrm{CD} 45 \mathrm{R} / \mathrm{B} 220^{+}$and $\mathrm{Gr}-1^{+}$cells had infiltrated into the tumor tissue of the SW1990/si-MUC5AC cells. Furthermore, cancer-associated antigen specific antibodies were detected at high levels in the sera from the SW1990/siMUC5AC cell-bearing mice. These results suggest that tumorassociated MUC5AC expressed on the surface of pancreatic
\end{abstract}

Correspondence to: Dr Tetsuji Sawada, Department of Surgical Oncology, Osaka City University Graduate School of Medicine, 1-4-3 Asahi-machi, Abeno-ku, Osaka 545-8585, Japan

E-mail: m1355299@med.osaka-cu.ac.jp

Key words: MUC5AC, mucin, glycoprotein, immunosuppression, short interfering RNA (siRNA), pancreatic cancer cancer cells supports the escape of pancreatic cancer cells from immunosurveillance. The present findings highlight a new dimension of MUC5AC as a functional immunosuppressive agent and its important role in pancreatic cancer progression.

\section{Introduction}

Mucins are heavily glycosylated proteins that are expressed in mucosal tissues, establish a selective molecular barrier at the epithelial surface, and engage in signal transduction pathways that regulate morphogenesis. Mucins can be classified into MUC1-20, which each have characteristic tandem repeat sequences. The amino acid residues of mucin are rich in serine and threonine, so have the potential to be O-glycosylated, and sugar chains constitute up to $80 \%$ of their molecular weight (1). In addition, mucins influence many cellular processes including growth, differentiation, transformation, adhesion, invasion, and immune surveillance. According to their cellular localization, mucins are divided into two classes: membrane bound mucins and secreted mucins. The secreted mucins, which lack a transmembrane domain and are secreted into extracellular spaces, include MUC2, MUC5AC, MUC5B, MUC6, MUC7, MUC8, and MUC19. The expression of secreted mucins is restricted to secretory organs and cell types. The membrane bound mucins, which are type I membrane proteins, include MUC1, MUC3, MUC4, MUC12, MUC13, MUC15, MUC16, MUC17, and MUC20. The membrane bound mucins are also considered to act as sensors of the external environment. Alterations in the gene expression of mucins accompany the development of cancer and influence cellular growth, differentiation, transformation, adhesion, and invasion (2-14). For example, aberrant expression of MUC4 in pancreatic, lung, breast, colon, and ovarian malignancies potentiates tumor cell growth and metastasis by altering the behavioral properties of tumor cells (15). MUC2-deficient mice showed increased proliferation, decreased apoptosis, and increased intestinal epithelial cell infiltration and frequently developed small intestinal, rectal, and gastrointestinal tumors (16). These results showed that MUC2 is involved in the suppression of cancer that protect the gastrointestinal tract. 
It has been reported that MUC5AC is aberrantly expressed in premalignant and malignant lesions as well as in several pancreatic cancer cell lines, but is undetectable in normal pancreatic tissue (17-21; Ho et al, Gastroenterology 118: abs. $664,2000)$. In our previous study, MUC5AC was expressed in $89.6 \%$ (60 of 67 cases) of pancreatic cancer cases (unpublished data). However, the details of how MUC5AC is involved in the process of malignant transformation, proliferation, and metastasis in pancreatic cancer remain to be elucidated. In the present study, we adapted the small interfering RNA (siRNA) technique to suppress MUC5AC expression in human pancreatic cancer cells and evaluated the effect of MUC5AC-knockdown on human pancreatic cancer cells in vitro and in vivo to clarify its functional role in human pancreatic cancer progression.

\section{Materials and methods}

Materials. The human pancreatic cancer cell lines SW1990, BxPC3, and PK-45P were purchased from Summit Pharmaceuticals International Corp. (Tokyo, Japan), Dainippon Sumitomo Pharma Biomedical Co. Ltd. (Osaka, Japan), and Cell Resource Center for Biomedical Research, Institute of Development, Aging and Cancer Tohoku University (Miyagi, Japan), respectively. The mouse anti-human MUC5AC monoclonal antibody (K-MAC5, IgG1) was developed in our laboratory. The rat anti-mouse CD45R/B220 monoclonal antibody (clone RA3-6B2) against B lymphocytes and the Ly-6G and Ly-6C (Gr-1, clone RB6-8C5) monoclonal antibody against granulocytes were purchased from Becton-Dickinson (San Jose, CA).

Mice. The experimental protocol was approved by the Ethics Committee on Animal Experiments of the Biomedical Research Laboratories of Kureha Corp., and the mice were treated in accordance with the guidelines of the committee. Five-week-old specific-pathogen-free female BALB/c-nu/nu mice purchased from Charles River Japan, Inc. (Kanagawa, Japan) were acclimatized for one week and then used in the experiments at the age of six weeks. The mice were allowed free access to sterilized CE-2 food (Oriental Yeast, Tokyo, Japan) and sterilized tap water and were bred at $25 \pm 2^{\circ} \mathrm{C}$, a humidity of $55 \pm 7 \%$, laminar flow, and under a 12-h light/12-h dark cycle at 150-300 lux. To maintain a uniform environment, noise was carefully avoided, and only the experimenters and keepers were allowed into the animal room.

Cell culture conditions. SW1990 cells were cultured in Dulbecco's modified Eagle's medium (DMEM; Invitrogen Corp., San Diego, CA) supplemented with $10 \%$ fetal bovine serum (FBS; Biowest, Nuaill, France), $50 \mathrm{IU} / \mathrm{ml}$ penicillin, and $50 \mu \mathrm{g} / \mathrm{ml}$ streptomycin. BxPC3 and PK-45P cells were cultured in Roswell Park Memorial Institute (RPMI)-1640 (Invitrogen) supplemented with 10\% FBS (Biowest), $50 \mathrm{IU} / \mathrm{ml}$ penicillin, and $50 \mu \mathrm{g} / \mathrm{ml}$ streptomycin. The cells were grown at $37^{\circ} \mathrm{C}$ under $5 \% \mathrm{CO}_{2}$ in a humidified atmosphere and passaged before they reached confluency using $0.25 \%(\mathrm{w} / \mathrm{v})$ trypsin solution containing $0.04 \%(\mathrm{w} / \mathrm{v})$ EDTA.

Construction of the siRNA expression vector. Eight types of siRNA sequences (19-mer) against human MUC5AC were designed using a computer algorithm and tested for silencing efficacy in transient assays. The MUC5AC siRNA target sequence 5'-TTTGAGAGACGAAGGATAC-3' was found to be the sequence showing the most marked effects and cloned in order to generate a stable siRNA expressing construct into the pSilencer 3.1-H1 neo vector (Ambion, Inc., Austin, TX). Briefly, oligonucleotides (64-mer) encoding 19-mer hairpin sequences specific to the mRNA target were designed. These contained two complementary domains (sense and antisense) separated by the loop sequence 5'-TTCAAGAGA-3'. Doublestranded oligonucleotides were ligated into the pSilencer 3.1H1 neo vector at BamHI (Takara Bio Inc., Shiga, Japan) and HindIII (TaKaRa) restriction sites (pSilencer/si-MUC5AC). The plasmid was then amplified in chemically competent Escherichia coli (DH5a cells).

Quantitative real-time PCR. Quantitative real-time PCR (qRT-PCR) was performed with a Light Cycler system (Roche Diagnostics K.K., Tokyo, Japan) using the Light Cycler DNA master SYBR-Green I kit (Roche Diagnostics K.K.) according to the manufacturer's protocol. The following primers were used: for human MUC5AC gene: 5'-GCCACCGCTGCGGC CTTCTTC-3' (forward) and 5'-GTGCACGTAGGAGGACA GCGC-3' (reverse) and for human glyceraldehyde-3-phosphate dehydrogenase (GAPDH) gene: 5'-GAAGGTGAAGGTCGG AGTC-3' (forward) and 5'-GAAGATGGTGATGGGAT TTC-3' (reverse). GAPDH gene expression was used for cDNA normalization. Amplification was carried out as follows: initial denaturation at $95^{\circ} \mathrm{C}$ for $15 \mathrm{~min}$, followed by 40 cycles at $95^{\circ} \mathrm{C}$ for $10 \mathrm{sec}$, a touchdown annealing $\left(0.5^{\circ} \mathrm{C} /\right.$ cycle $)$ from $68-60^{\circ} \mathrm{C}$ for $10 \mathrm{sec}$, and elongation at $72^{\circ} \mathrm{C}$ for $10 \mathrm{sec}$. After the completion of PCR amplification, a melting curve analysis was performed.

Transfection and selection of clones. SW1990 and BxPC3 cells were transfected with pSilencer/si-MUC5AC as a target vector or $\mathrm{pSilencer/si-mock}$ as a control vector using Genejuice (Merck, Darmstadt, Germany) according to the manufacturer's instructions. Briefly, the cells were seeded in a $100-\mathrm{mm}$ culture dish and grown to $60-80 \%$ confluence. The medium was then removed, and the cells were washed twice in serum-free medium, before being incubated for $8 \mathrm{~h}$ in $15 \mathrm{ml}$ serum-free medium with $18 \mu \mathrm{l}$ Genejuice and $6 \mu \mathrm{g}$ plasmid DNA. The cells were then washed in medium and cultured for $24 \mathrm{~h}$ in complete medium, and each cell was subcultured after $48 \mathrm{~h}$ ( $\sim 5$-fold). SW1990 and BxPC3 cells were selected by culturing them in the presence of geneticin at 600 and $400 \mu \mathrm{g} / \mathrm{ml}$, respectively. The efficiency of MUC5AC-knockdown was tested by qRT-PCR and FACS analysis.

Fluorescence-activated cell sorting (FACS) analysis. The cells were detached using $0.04 \%(\mathrm{w} / \mathrm{v})$ EDTA, washed with PBS, and resuspended in PBS containing $2 \% \mathrm{FBS}$ and $2 \mathrm{mM}$ EDTA (FACS buffer) and then were incubated with an appropriate dilution of K-MAC5 $(1 \mu \mathrm{g})$, mouse serum (100-fold dilution), or isotype control $(1 \mu \mathrm{g})$ per $10^{6}$ cells for $2 \mathrm{~h}$ at $4^{\circ} \mathrm{C}$. After being washed three times in FACS buffer, the cells were incubated with a FITC-conjugated rabbit antimouse secondary antibody (Becton-Dickinson) for $30 \mathrm{~min}$ at $4^{\circ} \mathrm{C}$. The cells were then washed three times and resuspended 
in FACS buffer, before being analyzed on a FACScan (BectonDickinson) with $>10^{4}$ cells analyzed per sample. The data files were analyzed using CellQuest (Becton-Dickinson), and the cells were gated using forward and side scatter variables to eliminate dead cells and debris.

Cell proliferation assay. The cells were suspended in each medium at a density of $1 \times 10^{4} / \mathrm{ml}$, and $100 \mu \mathrm{l}$ suspensions were seeded in 96-well plates. After incubation for $0,1,2$, 3 , or 4 days, $20 \mu 1$ of 3-(4,5-dimethylthiazol-2-yl)-2,5diphenyltetrazolium bromide (MTT) solution $(5 \mathrm{mg} / \mathrm{ml})$ was added. Following incubation at $37^{\circ} \mathrm{C}$ for $3 \mathrm{~h}$, the supernatant was removed, and dimethylsulfoxide (DMSO) was added at $100 \mu \mathrm{l} /$ well. The absorbance of formazan was measured at a wavelength of $570 \mathrm{~nm}$ and a reference wavelength of $630 \mathrm{~nm}$ with a Bio-Rad Microplate Reader 550 (Bio-Rad Laboratories Inc. Tokyo, Japan). Cell growth curves were plotted from the mean \pm standard deviation (SD).

Tumorigenicity assay in xenograft models. Exponentially growing cells were detached with $0.25 \%(\mathrm{w} / \mathrm{v})$ trypsin solution containing $0.04 \%(\mathrm{w} / \mathrm{v})$ EDTA and resuspended in PBS at a density of $1 \times 10^{8} / \mathrm{ml}$. Each cell line $\left(1 \times 10^{7} / \mathrm{mice}\right)$ was implanted s.c. into the flanks of nude mice, and their body weight and tumor volume were measured at least once a week. To determine tumor volume, two bisecting diameters were measured using a slide caliper, and the tumor volumes were calculated using the following formula: tumor volume $=$ length $\mathrm{x}(\text { width })^{2}$ $x 0.5236$ (22). At the end of the experiment, the tumors were removed, weighed, and fixed in $10 \%$ formaldehyde neutral buffer solution. Ten mice were used for each cell line. Tumors growth curves were plotted as the mean volume \pm standard error (SE).

Metastasis assay. The experimental lung colonization was evaluated by injecting $1 \times 10^{6}$ viable cells in $100 \mu 1$ of PBS i.v. into the lateral tail vein of the BALB/c-nu/nu mice. The mice were euthanized on day 30 posttransplantation, their lungs were removed, and the total number of surface colonies was estimated under a dissecting microscope. Ten mice were used for each cell line.

Histology and immunohistochemistry. Lymphocyte infiltration into tumor tissue was evaluated by immunohistochemical analysis. Tumor tissues fixed in $10 \%$ formaldehyde neutral buffer solution were embedded in paraffin. Paraffin-embedded sections of $4 \mu \mathrm{m}$ thickness were deparaffinized with xylene and ethanol, treated with $3 \%$ hydrogen peroxide solution for $20 \mathrm{~min}$ to inactivate endogenous peroxidase, and blocked with $2 \%$ porcine plasma in TBS for 15 min to reduce non-specific binding. Then, K-MAC5 monoclonal antibody, rat anti-mouse CD45R/B220 and Gr-1 monoclonal antibodies were added, and the sections were incubated at room temperature for $1 \mathrm{~h}$. After being washed three times with TBS containing $0.05 \%$ Tween-20 (TBS-T), a horseradish peroxidase-labeled secondary antibody was added, and the sections were incubated at room temperature for $30 \mathrm{~min}$. After being washed a further three times with TBS-T, immunoreaction was visualized with Liquid DAB Chromogen (Dako Japan Co. Ltd., Kyoto, Japan), and the sections were counterstained with hematoxylin. After being mounted on a glass slide with Entellan Neu (Merck), the stained specimens were examined using a microscope. In parallel to the immunostaining, hematoxylin and eosin (H\&E) staining was performed for morphological evaluation.

Secondary tumor challenge. The primary tumor (SW1990/siMUC5AC cells) was implanted via the injection of $1 \times 10^{7}$ cells into the right flanks of nude mice. After 42 days, a secondary tumor (SW1990/si-mock cells) was implanted on the opposite flank. Tumor growth was measured at least once a week according to the above method.

Statistical analysis. All data are expressed as means \pm SD or SE. Statistical significance was determined by the Student's t-test. P-values $<0.05$ were considered significant.

\section{Results}

Establishment of MUC5AC-knockdown cells by siRNA. The MUC5AC-overexpressed human pancreatic cancer cell lines SW1990 and BxPC3 were transfected with the pSilencer 3.1-H1 neo/si-MUC5AC vector or the pSilencer 3.1-H1 neo/simock vector as a negative control, and stable transfectants were established. In order to confirm the silencing efficiency of MUC5AC siRNA, the MUC5AC mRNA and protein levels in the transfectants were determined by qRT-PCR and FACS analysis. qRT-PCR data showed that MUC5AC expression was significantly decreased in stably transfected cells containing MUC5AC siRNA (SW1990/si-MUC5AC and BxPC3/si-MUC5AC cells) compared with si-mock cells containing the control vector (SW1990/si-mock and BxPC3/si-mock cells) (Fig. 1A). The loss of MUC5AC from the cell surface was determined by FACS analysis. It was found that the surface expression of MUC5AC was also substantially down-regulated in SW1990/si-MUC5AC and BxPC3/si-MUC5AC cells (si-MUC5AC transfectants) compared with the SW1990/si-mock and BxPC3/si-mock cells (si-mock transfectants), respectively (Fig. 1B). However, there were no differences between the si-mock transfectants and parental cells with regard to their mRNA and protein expression levels (data not shown). Therefore, in the following experiments, SW1990/si-mock and BxPC3/si-mock cells were used as negative controls.

In vitro cell survival, proliferation, and morphology. To investigate the effect of MUC5AC on cell proliferation, SW1990/si-mock, SW1990/si-MUC5AC, BxPC3/si-mock, and $\mathrm{BxPC} 3 / \mathrm{si}-\mathrm{MUC} 5 \mathrm{AC}$ cells were seeded in 96-well plates and the number of cells was determined over time. As shown in Fig. 2A and B, no difference in cell proliferation was observed between the SW1990/si-MUC5AC and SW1990/simock cells or between the BxPC3/si-MUC5AC and BxPC3/simock cells. Next, we examined whether the knockdown of MUC5AC was associated with any morphological changes. Each cell was plated, and morphological changes were assessed during proliferation from sparsity to confluence. As a result, the SW1990/si-MUC5AC and BxPC3/si-MUC5AC cells tended to cluster together, and some of them exhibited a fibroblast-like morphology and a tightly packed cobblestonelike morphology, respectively (Fig. 2C), but the difference in 
A

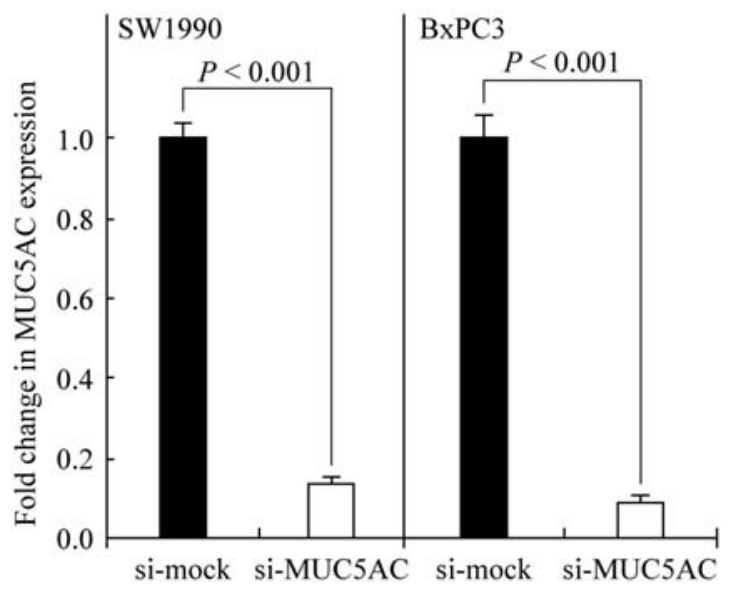

B
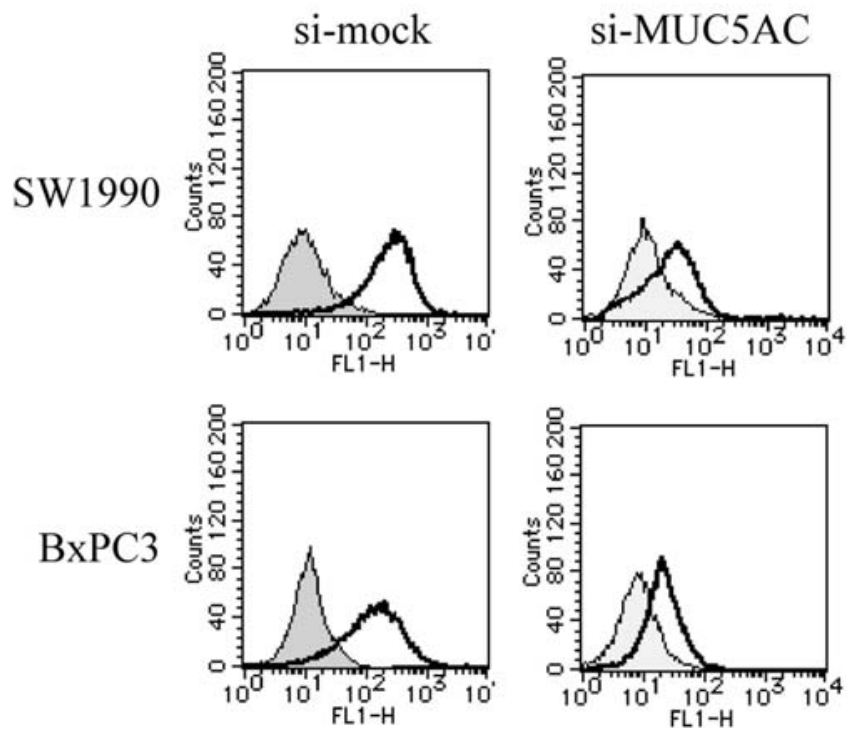

Figure 1. Establishment of MUC5AC-knockdown human pancreatic cancer cell lines. SW1990 and BxPC3 cells were stably transfected with pSilencer 3.1-H1 neo/si-mock or pSilencer 3.1-H1 neo/si-MUC5AC (an MUC5AC specific siRNA expression vector). The MUC5AC expression of each cell was then analyzed by qRT-PCR and FACS as described in Materials and methods. (A) MUC5AC and GAPDH mRNA expression levels were evaluated by qRT-PCR. qRT-PCR was performed on cDNA from each cultured cells using human MUC5AC-specific primers. Values were normalized using the internal control gene, GAPDH. All PCR experiments were performed in triplicate. Columns, mean of triplicate determinations; bars, SD. (B) FACS analysis of MUC5AC expression in mock- and MUC5AC-knockdown cells. Fluorescence of MUC5AC expressing SW1990 and BxPC3 cells probed with K-MAC5 mAb. Top, SW1990/si-mock and SW1990/si-MUC5AC cells; bottom, BxPC3/si-mock and BxPC3/si-MUC5AC cells; gray filled histograms, isotypic control antibody; bold lines, K-MAC5. The experiment was repeated twice. Representative data are shown.

morphology between the si-mock and si-MUC5AC cells was not marked.

In vivo tumorigenicity and metastatic potential. As both the si-mock and si-MUC5AC cells showed exactly the same proliferation rate in vitro, we examined their ability to induce tumorigenicity in vivo. SW1990/si-MUC5AC cell-challenged mice barely developed any visible solid tumors, but the si-mock
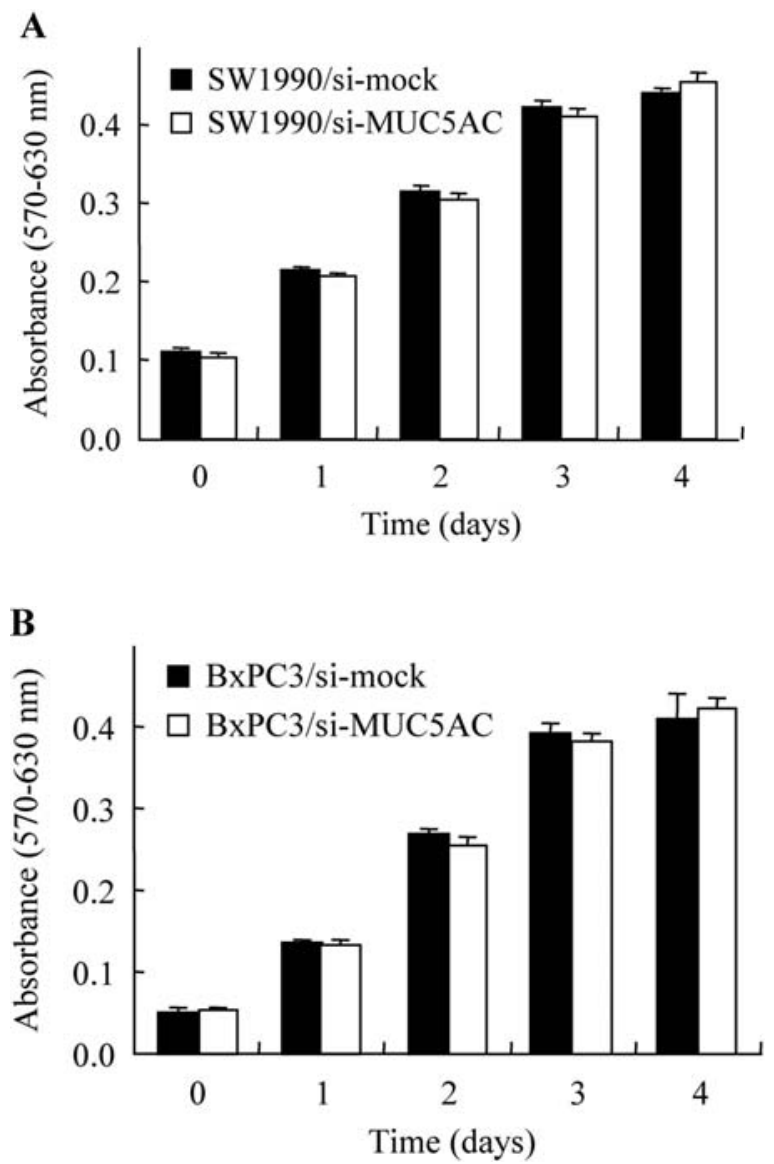

$\mathbf{C}$

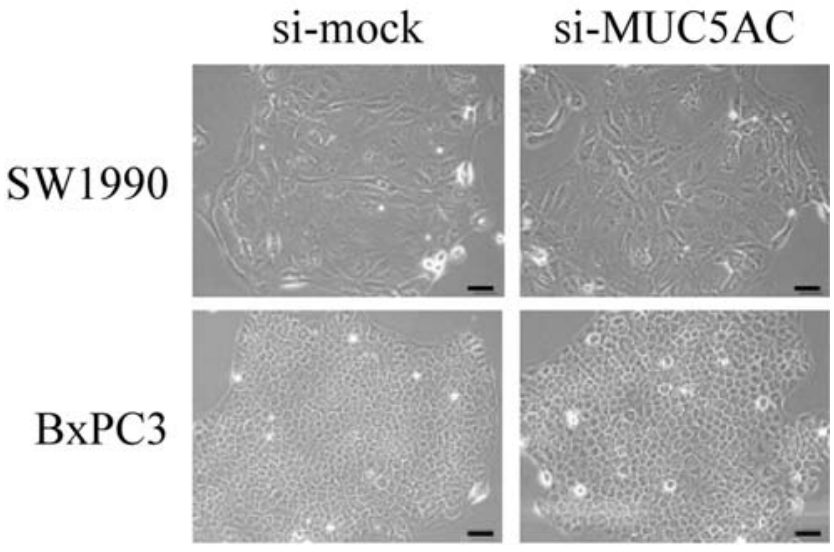

Figure 2. Effect of MUC5AC on cell growth and morphologic change. SW1990/si-mock and SW1990/si-MUC5AC (A) or BxPC3/si-mock and BxPC3/si-MUC5AC cells (B) seeded in a 96-well plate were cultured for 0 , 1, 2, 3, or 4 days. Filled columns, si-mock cells; open columns, si-MUC5AC cells. Their cell number was determined by their absorbance as described in Materials and methods. The experiment was repeated thrice. Representative data are shown. Columns, mean of triplicate determinations; bars, SD. SW1990/si-mock and SW1990/si-MUC5AC (C, top) or BxPC3/si-mock and $\mathrm{BxPC} 3 /$ si-MUC5AC cells $(\mathrm{C}$, bottom) were cultured during proliferation from sparsity to confluence, and phase-contrast microscopic images were observed. The experiment was repeated thrice. Data shown are representative images captured from one of three independent experiments performed in triplicate. Bar, $50 \mu \mathrm{m}$.

cell-challenged mice had developed tumors by one week as expected (Fig. 3A). The tumor growth of the SW1990/siMUC5AC cells was suppressed compared to that of the 

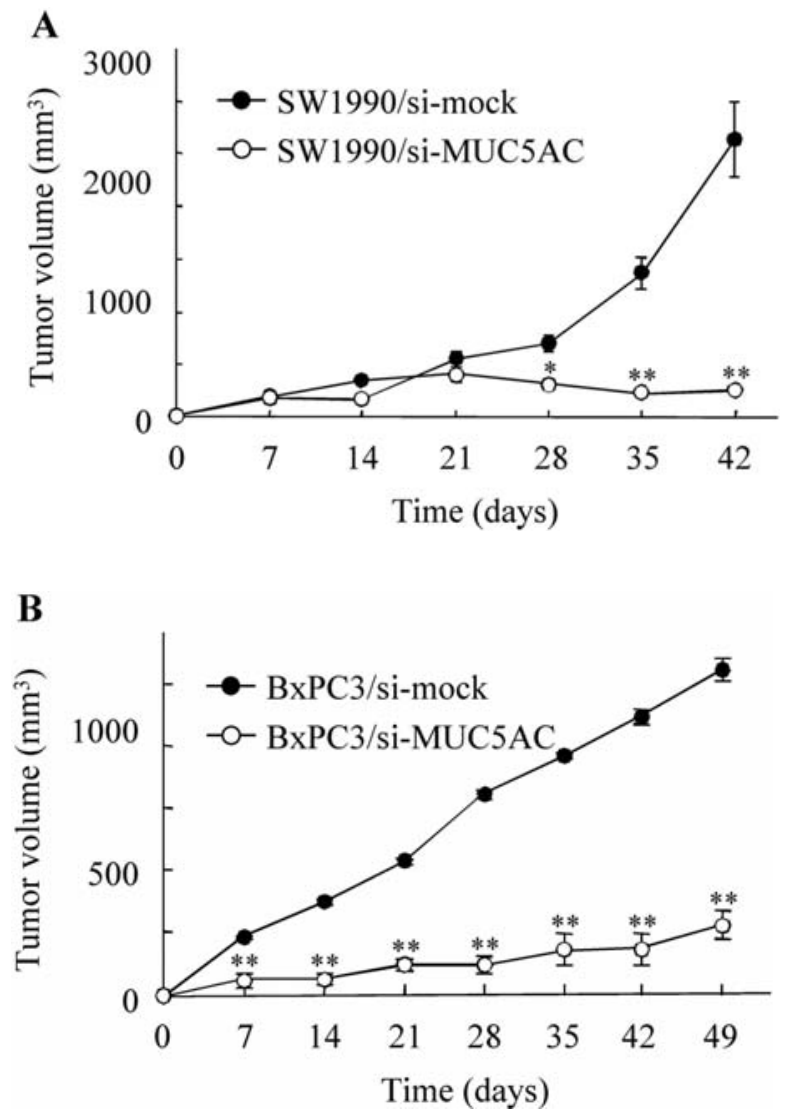

Figure 3. MUC5AC-knockdown by siRNA significantly inhibited tumor xenograft growth. (A) Tumor growth curves of SW1990/si-mock (filled circles) and SW1990/si-MUC5AC (open circles) cells. (B) Tumor growth curves of BxPC3/si-mock (filled circles) and BxPC3/si-MUC5AC (open circles) cells. The cells were s.c. implanted with $1 \times 10^{7}$ cells into the nude mice on day 0 . The mice were monitored for tumor formation until 42 (A) and 49 (B) days, respectively, and the tumors were measured on the indicated days. These experiments were repeated at least thrice. Representative data are shown. Points, mean tumor volume of 10 mice for each group; bars, SE. Statistically significant at ${ }^{*} \mathrm{P}<0.005$ and ${ }^{* *} \mathrm{P}<0.001$ (versus si-mock cellchallenged mice group).

SW1990/si-mock cells after day $28(\mathrm{P}<0.005)$. Also tumor volumes were significantly increased in the mice injected with $\mathrm{BxPC} 3 / \mathrm{si}$-mock cells compared with the tumor growth in those injected with BxPC3/si-MUC5AC cells (Fig. 3B). The tumor growth of the BxPC3/si-MUC5AC cells was decreased compared with that of the BxPC $3 /$ si-mock cells after day 7 $(\mathrm{P}<0.001)$. Furthermore, to examine whether the proliferation of si-MUC5AC cells was also inhibited in a lung metastasis model, the SW1990/si-mock and SW1990/si-MUC5AC cells were injected i.v. into nude mice, and metastases were enumerated after day 30 . As a result, there were no lung metastases that could be confirmed under a dissecting microscope in the SW1990/si-MUC5AC cell-challenged mice, while many metastases were observed in the SW1990/si-mock cell-challenged mice (Table I).

Accumulation of immunocytes in the tumor tissues. To identify the types of immunocytes that infiltrated into the tumor tissues, the tumor challenge sites were removed and subjected to histological and immunohistochemical examination. First, MUC5AC expression at the SW1990/si-mock cell-challenged
Table I. MUC5AC-knockdown in tumor cells significantly diminishes lung metastasis. ${ }^{\mathrm{a}}$

Count of lung metastases

\begin{tabular}{lrrrrrrrrrr}
\hline Mice no. & 1 & 2 & 3 & 4 & 5 & 6 & 7 & 8 & 9 & 10 \\
$\begin{array}{l}\text { SW1990/ } \\
\text { si-mock }\end{array}$ & 4 & 10 & 3 & 5 & 6 & 8 & 7 & 6 & 10 & 4 \\
$\begin{array}{l}\text { SW1990/ } \\
\text { si-MUC5AC }\end{array}$ & 0 & 0 & 0 & 0 & 0 & 0 & 0 & 0 & 0 & 0 \\
\hline
\end{tabular}

${ }^{\text {a Tumor cells }}\left(1 \times 10^{6}\right)$ were injected into the lateral tail veins of the mice. The lungs were collected 30 days after posttransplantation, and the total numbers of surface colonies were counted. Ten mice were used for each cell line. The experiment was repeated twice. Representative data are shown.

site and SW1990/si-MUC5AC cell-challenged site was examined by IHC using K-MAC5 monoclonal antibody, and its significant suppression at the latter site was confirmed (Fig. 4A). Next, rat anti-mouse CD45R/B220 and Gr-1 monoclonal antibodies were used for the detection of the $\mathrm{B}$ lymphocytes and granulocytes, respectively. As shown in Fig. 4B, H\&E staining showed that many immunocytes had infiltrated into the SW1990/si-MUC5AC cell-challenged site. Immunohistochemistry revealed that the infiltrating cells were CD45R/B220+ (Fig. 4C) and Gr-1+ cells (Fig. 4D) that were scattered throughout the tumor, but were particularly localized in the epithelial and surrounding stromal compartments. In contrast, few or no infiltrating immunocytes were found at the SW1990/si-mock cell-challenged site.

Antibody production by B lymphocytes. As it was shown in the above immunostaining examination that many B lymphocytes had accumulated at the SW1990/si-MUC5AC cell-challenged site, we investigated whether B lymphocytes raised specific antibodies against the implanted tumor cells. To do this, sera were collected from SW1990/si-mock and SW1990/siMUC5AC cell-challenged mice and were used to evaluate the responsiveness to human pancreatic PK-45P cells, which do not express MUC5AC, by FACS analysis. FACS analysis showed that the sera of the SW1990/si-MUC5AC cellchallenged mice more effectively recognized PK-45P cells as a foreign substance, compared to that of the SW1990/simock cell-challenged mice (Fig. 4E).

Rejection of a secondary challenge with SW1990/si-mock cells in mice subjected to primary challenge with SW1990/siMUC5AC cells. It was thought that memory B cells may play a pivotal role in the rejection of grafted SW1990/si-MUC5AC cells in nude mice. So, we investigated whether a secondary challenge with SW1990/si-mock cells would be rejected in mice that had previously been challenged with SW1990/ si-MUC5AC cells. As a first step, SW1990/si-MUC5AC cells (the primary tumor) were implanted into the right flanks of nude mice. After 42 days, SW1990/si-mock cells 

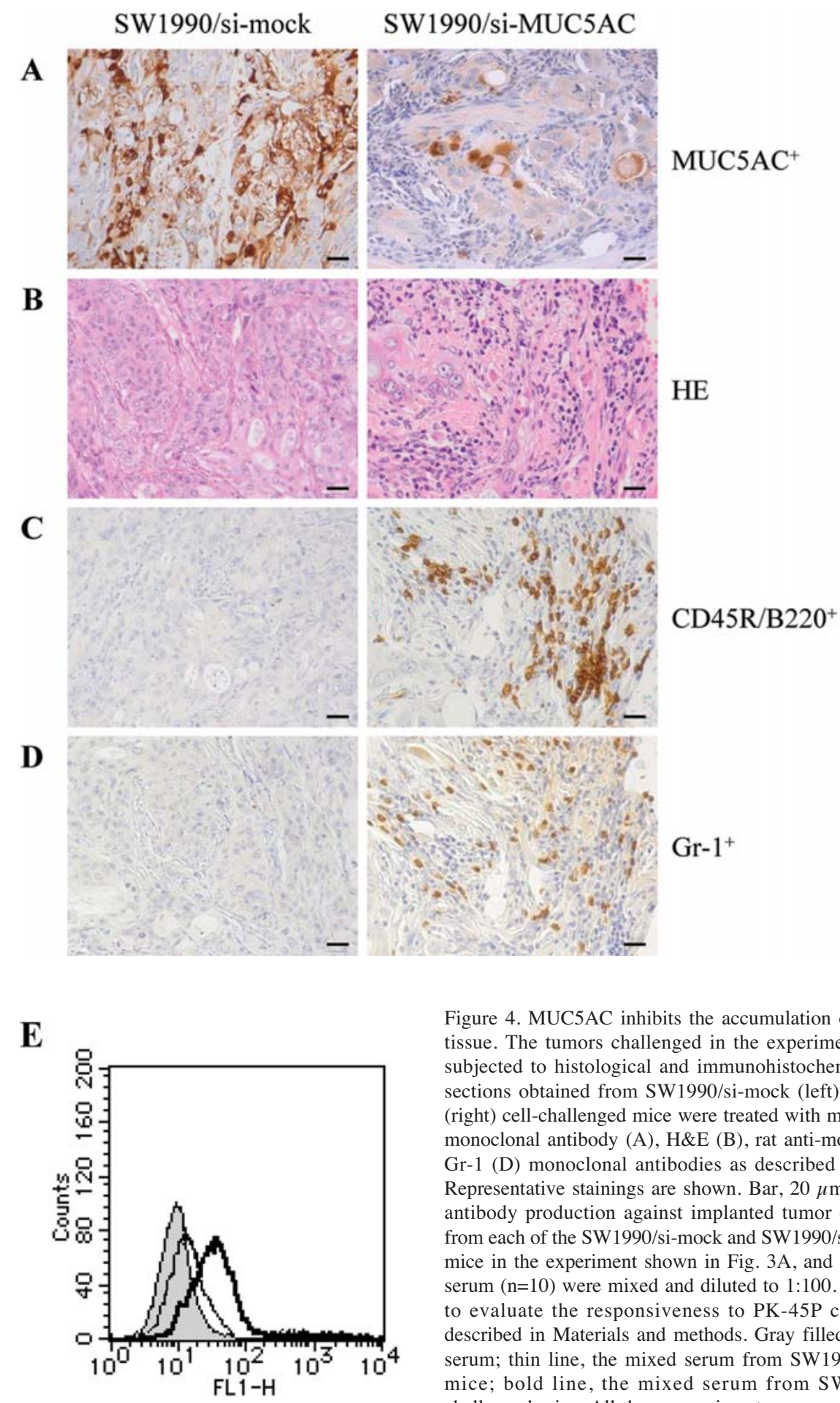

Figure 4. MUC5AC inhibits the accumulation of immunocytes into tumor tissue. The tumors challenged in the experiment shown in Fig. 3A were subjected to histological and immunohistochemical examinations. Tumor sections obtained from SW1990/si-mock (left) and SW1990/si-MUC5AC (right) cell-challenged mice were treated with mouse anti-human MUC5AC monoclonal antibody (A), H\&E (B), rat anti-mouse CD45R/B220 (C), and Gr-1 (D) monoclonal antibodies as described in Materials and methods. Representative stainings are shown. Bar, $20 \mu \mathrm{m}$. (E) MUC5AC suppresses antibody production against implanted tumor cells. Serum was collected from each of the SW1990/si-mock and SW1990/si-MUC5AC cell-challenged mice in the experiment shown in Fig. 3A, and equivalent amounts of each serum $(n=10)$ were mixed and diluted to 1:100. The mixed serum was used to evaluate the responsiveness to PK-45P cells by FACS analysis as described in Materials and methods. Gray filled histogram, normal control serum; thin line, the mixed serum from SW1990/si-mock cell-challenged mice; bold line, the mixed serum from SW1990/si-MUC5AC cellchallenged mice. All these experiments were performed at least thrice. One representative data set of three independent experiments is shown.

(the secondary tumor) were implanted into the opposite flank (Fig. 5A). As a result, the secondary tumor growth of SW1990/ si-mock cells was significantly decreased in the primary tumor-bearing mice compared with the primary tumor-absent mice (Fig. 5B). Significant inhibition was shown after day 7 of the secondary challenge $(\mathrm{P}<0.001)$. In other words, the primary graft of SW1990/si-MUC5AC cells caused strong rejection of the secondary tumor graft. Furthermore, the primary tumor no longer developed and was not promoted by the secondary inoculation (data not shown). In contrast, the

tumor volumes of the SW1990/si-mock cells in the primary tumor-absent mice were significantly increased, as expected from the results shown in Fig. 3A.

\section{Discussion}

In spite of recent developments that have improved the prevention, screening, and therapy for pancreatic cancer, it still has a poor prognosis: a 5-year survival rate of up to $3 \%$ and a median survival of up to 6-month. This poor prognosis 
A
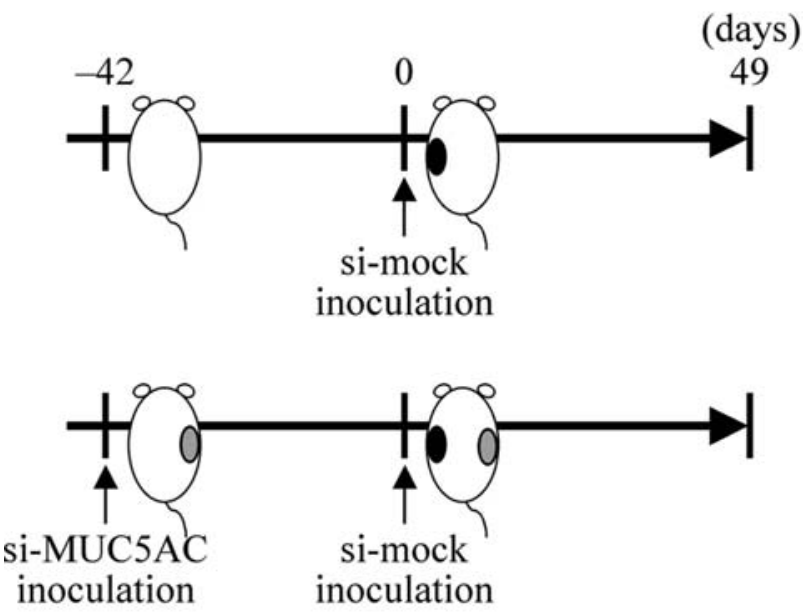

SW1990/si-mock
O SW1990/si-MUC5AC

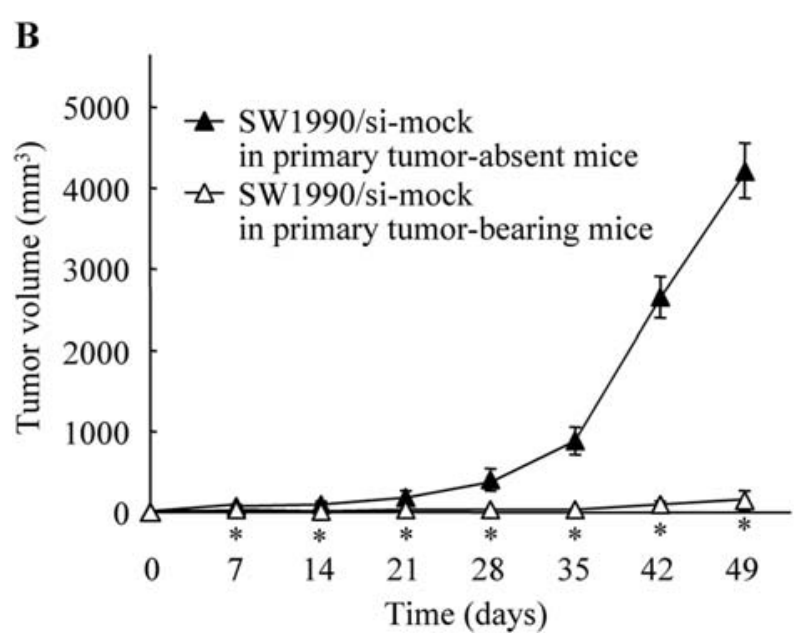

Figure 5. MUC5AC suppresses the immune memory system. (A) Animal experiment protocol. (B) Tumor volume. The primary tumor (SW1990/siMUC5AC cells) was implanted with $1 \times 10^{7}$ cells into the flanks of nude mice. After 42 days, a secondary tumor (SW1990/si-mock cells) was implanted with $1 \times 10^{7}$ cells into the primary tumor-absent mice (filled triangles) and the primary tumor-bearing mice (open triangles). Tumor size was measured on the indicated days. These experiments were repeated at least thrice. Representative data are shown. Points, mean tumor volume of 10 mice for each group; bars, SE. Statistically significant at ${ }^{*} \mathrm{P}<0.001$ (versus primary tumor-absent mice group).

is a consequence of metastatic disease, which results from a lack of early detection and effective treatment. Concerning the relationship between the malignancy grade of pancreatic cancer and mucins, it was previously reported that MUC1 and carbohydrate antigens such as sialyl Lewis antigen are related to metastatic potential and prognosis. For example, Tsutsumida et al previously reported that down-regulation of MUC1 expression by siRNA significantly decreased human pancreatic cancer cell proliferation in vitro and in vivo (23). Chaturvedi et al demonstrated that silencing MUC4 expression by transfecting MUC4-specific short hairpin RNA into a human pancreatic cell line decreased cell growth and metastasis in vivo and induced ectopic expression of growth- and metastasis-associated genes. They also showed that overexpression of MUC4 is associated with increased tumor cell growth and metastasis via interference with the interaction between cancer cells and extracellular matrix proteins (24).

MUC5AC has been shown to be a major component of the stomach and airway, and little or no expression of MUC5AC is found in other normal tissues; whereas, it is anomalously expressed in human pancreatic and colorectal cancers. This implies that MUC5AC plays an important role in these cancers, but few reports exist on its function. Here, we established MUC5AC-knockdown cells by introducing siRNA and investigated how MUC5AC contributes to tumor progression. The findings from the present study showed that the ectopic expression of MUC5AC was not involved in cell growth in vitro, but that it was intimately involved in tumor growth in vivo. The expression levels of cell-adhesion factors, some of which, such as sialylLewis $^{\mathrm{a}}$, sialyl-Lewis ${ }^{\mathrm{x}}$, and E-cadherin are known to be related to metastasis, were not affected in si-MUC5AC cells (data not shown). In addition, to test the alteration in migratory capacity after the suppression of MUC5AC, we performed a scratch wound-healing assay in si-MUC5AC and si-mock cells. The scratch wound-healing assay is considered to be an in vitro model of the epithelial cell migration that occurs during wound healing. The scratch wounds in both cells were closed with a large portion after $48 \mathrm{~h}$ and no significant delay in the migration of si-MUC5AC cells into the empty space was observed (data not shown). Therefore, it appears that the functions of MUC5AC in pancreatic cancer are essentially different from those of other mucins. In the future, we are going to perform a microarray analysis of si-mock and siMUC5AC cells and to investigate the expression of tumorassociated genes.

An analysis of the functional role of MUC5AC was reported for colorectal cancer. It may be assumed that the expression of MUC5A plays a role in the invasiveness of cancers, as it is detected in the early stages of human colorectal cancer. Truant et al reported on the invasive properties of the human colon carcinoma cell line HT-29 (HT-29 STD) and its highly MUC5AC secreting-variant (HT-29 5M21). It was found that HT-29 STD cells are non-invasive; whereas, HT-29 $5 \mathrm{M} 21$ cells are invasive due to a lack of cell-cell adhesion through E-cadherin, and HT-29 STD cells and HT-29 5M21 cells also have different morphologies, the former grows in multilayers, but the latter grows in monolayers (25). In the present study, MUC5AC-knockdown cells and their parental cells proliferated in multilayers and did not differ in morpho$\operatorname{logy}$ (Fig. 2). These results indicate that the MUC5AC molecules of pancreatic and colorectal cancers may have different functions.

In a previous report, mucins were found to have inhibitory effects on the antitumor response of the host and to promote tumor progression and metastasis. Cell-cell adhesion contributes to the normal development and function of cells; whereas, the highly glycosylated rigid structure of the mucins reduces both homotypic cell-cell adhesion and cell-substratum interactions (26). Almost all of the functions of mucins reported until now have been connected to proliferation, adhesion, migration, or invasion, and in vitro as well as in vivo proliferation has been reported to be inhibited by knockdown 
of the expression of mucins. However, the present study demonstrated that MUC5AC-knockdown affected in vivo proliferation but not in vitro proliferation, which differs from the results of previous reports. As shown in Figs. 2 and 3, MUC5AC-knockdown cells showed similar results to the control cells with regard to cell survival, proliferation, and morphology in vitro. However, in in vivo xenograft studies, their tumor growth was significantly inhibited compared with that of the control cells. So, why were in vivo tumorigenicity and metastasis inhibited when no marked in vitro effect was observed? One of the reasons for this inhibitory effect is thought to be induced by antibody-dependent cell-mediated cytotoxicity (ADCC). In the present study, B cells and neutrophils were found to accumulate at SW1990/si-MUC5AC cell-challenged sites (Fig. 4). Moreover, a greater number of antibodies against human pancreatic cells were present in the blood of the SW1990/si-MUC5AC cell-challenged mice compared to the levels produced by the SW1990/si-mock cell-challenged mice (Fig. 4E). Neutrophils are non-specific cytotoxic immunocytes that exclude infecting organisms. Recently, it has been reported that neutrophils also have antitumor effects mediated through ADCC via phagocytosis, elastase, or superoxide (27-29). ADCC is triggered by tumorspecific antibodies and $\mathrm{Fc}$ receptor-expressing cytolytic cells such as NK cells, neutrophils, eosinophils, and monocytes/ macrophages (30). According to these results, the present in vivo tumor inhibition was supposed to be induced by ADCC. Moreover, si-MUC5AC cells are a MUC5AC-knockdown cell line and are not able to grow in vivo. The SW1990/ si-MUC5AC cell-challenged mice used in this study significantly rejected a secondary tumor challenge with SW1990/simock cells, which are usually able to grow in vivo (Fig. 5B). As nude mice were used in the present experiments, antigen memory via cytotoxic $\mathrm{T}$ cells can not be involved. Therefore, the mice that received the si-MUC5AC cells might have produced specific antibodies against an unknown pancreatic cancer specific antigen, and these antibodies may have inhibited the secondary-challenged si-mock tumor via ADCC. It would be interesting to know which molecule serves as the antigen in this case, as its identification and analysis would provide a target for molecular pancreatic cancer therapeutics.

Another possible reason for the in vivo inhibition of tumorigenicity is that MUC5AC is directly involved in immune suppression and avoidance. Several reports have investigated MUC1 or MUC2. Overexpression of MUC1 in pancreatic cancer is associated with poor prognosis (31), and there is disputed evidence that tumors associated with MUC1 directly influence the response of immunocytes. For example, MUC1 inhibits the function of effector cells such as natural killer cells, lymphokine-activated killer cells, and cytotoxic T-cells. Also, soluble MUC1 induces apoptosis of activated T cells and inhibits the proliferation of natural killer cells and cytotoxic T-cells $(32,33)$. MUC2 has been reported to bind to a scavenger receptor of monocytes/macrophages and leads to the overproduction of PGE2, which suppresses immunocytes through COX2 $(34,35)$. In this study, the growth of MUC5AC-knockdown cells was inhibited in not only xenografted tumors but also a lung metastasis mode (Table I). Thus, it has been suggested that MUC5AC may be involved in immunosuppression and evasion from the immune system similarly to MUC1 and MUC2. However, future studies are needed to investigate the immunosuppressive mechanism of MUC5AC in human pancreatic cancer.

In conclusion, we investigated the role of MUC5AC in human pancreatic cancer progression using specific siRNA against MUC5AC. Our data showed that suppression of MUC5AC in two human pancreatic cancer cell lines dramatically reduced in vivo tumor growth and metastasis without affecting cell growth in vitro. Also infiltration of CD45R/B220 and $\mathrm{Gr}-1^{+}$cells was observed in tumor tissue generated by implanting si-MUC5AC cells into mice. Furthermore, specific antibodies against the tumor cells were mostly observed in the sera of SW1990/si-MUC5AC cell-bearing mice. These results suggested that the MUC5AC expressed on the surface of pancreatic cancer cells aids cancer cell escape from the immune system. The present findings highlight a new dimension of MUC5AC as a functional immunosuppressive agent and its important role in pancreatic cancer progression. Also, MUC5AC may be an important indicator for the diagnosis and prognosis of pancreatic cancer, and disruption of MUC5AC may have potential as a treatment for MUC5ACexpressing pancreatic and other cancers.

\section{Acknowledgements}

This work was conducted at Kureha Corporation, Tokyo, Japan and Osaka City University, Osaka, Japan. Hirotaka Hoshi, Motoyuki Uchida, Hikaru Saito, Hiroko Iijima, Mikako Toda-Agetsuma, and Tsutomu Wada are employees of Kureha Corporation, but the study was conducted with scientific integrity and presents no conflict of interest.

\section{References}

1. Carlstedt I, Sheehan JK, Corfield AP and Gallagher JT: Mucus glycoproteins: a gel of a problem. Essays Biochem 20: 40-76, 1985.

2. Bobek LA, Tsai H, Biesbrock AR and Levine MJ: Molecular cloning, sequence, and specificity of expression of the gene encoding the low molecular weight human salivary mucin (MUC7). J Biol Chem 268: 20563-20569, 1993.

3. Gum JR Jr, Hicks JW, Swallow DM, et al: Molecular cloning of cDNA derived from a novel human intestinal mucin gene. Biochem Biophys Res Commun 171: 407-415, 1990.

4. Gum JR Jr, Hicks JW, Toribara NW, Rothe EM, Lagace RE and Kim YS: The human MUC2 intestinal mucin has cysteinerich subdomains located both upstream and downstream of its central repetitive region. J Biol Chem 267: 21375-21383, 1992.

5. Gum JR Jr, Hicks JW, Toribara NW, Siddiki B and Kim YS: Molecular cloning of human intestinal mucin (MUC2) cDNA. Identification of the amino terminus and overall sequence similarity to prepro-von Willebrand factor. J Biol Chem 269: 2440-2446, 1994.

6. Gum JR Jr, Crawley SC, Hicks JW, Szymkowski DE and Kim YS: MUC17, a novel membrane-tethered mucin. Biochem Biophys Res Commun 291: 446-475, 2002.

7. Hollingsworth MA and Swanson BJ: Mucins in cancer: protection and control of the cell surface. Nat Rev Cancer 4: 45-60, 2004.

8. Moniaux N, Nollet S, Porchet N, Degand P, Laine A and Aubert JP: Complete sequence of the human mucin MUC4: a putative cell membrane-associated mucin. Biochem J 338: 325-333, 1999.

9. Fauquette V, Aubert S, Groux-Degroote S, Hemon B, Porchet N, van Seuningen I and Pigny P: Transcription factor AP-2 represses both the mucin MUC4 expression and pancreatic cancer cell proliferation. Carcinogenesis 28: 2305-2312, 2007. 
10. Porchet N, Pigny P, Buisine MP, Debailleul V, Degand P, Laine A and Aubert JP: Human mucin genes: genomic organization and expression of MUC4, MUC5AC and MUC5B. Biochem Soc Trans 23: 800-805, 1995.

11. Shankar V, Pichan P, Eddy RL Jr, et al: Chromosomal localization of a human mucin gene (MUC8) and cloning of the cDNA corresponding to the carboxy terminus. Am J Respir Cell Mol Biol 16: 232-241, 1997.

12. Toribara NW, Roberton AM, Ho SB, et al: Human gastric mucin. Identification of a unique species by expression cloning. J Biol Chem 268: 5879-5885, 1993.

13. Williams SJ, Wreschner DH, Tran M, Eyre HJ, Sutherland GR and McGuckin MA: Muc13, a novel human cell surface mucin expressed by epithelial and hemopoietic cells. J Biol Chem 276: 18327-18336, 2001.

14. Yin BW and Lloyd KO: Molecular cloning of the CA125 ovarian cancer antigen: identification as a new mucin, MUC16. J Biol Chem 276: 27371-27375, 2001 .

15. Bafna S, Singh AP, Moniaux N, Eudy JD, Meza JL and Batra SK: MUC4, a multifunctional transmembrane glycoprotein, induces oncogenic transformation of NIH3T3 mouse fibroblast cells. Cancer Res 68: 9231-9238, 2008.

16. Velcich A, Yang W, Heyer J, et al: Colorectal cancer in mice genetically deficient in the mucin Muc2. Science 295: 1726-1729, 2002.

17. Balague C, Gambus C, Carrato N, Porchet N, Aubert JP, Kim YS and Real FX: Altered expression of MUC2, MUC4, and MUC5 mucin genes in pancreas tissues and cancer cell lines. Gastroenterology 106: 1054-1061, 1994.

18. Voynow JA, Young LR, Wang Y, Horger T, Rose MC and Fischer BM: Neutrophil elastase increases MUC5AC mRNA and protein expression in respiratory epithelial cells. Am J Physiol 276: L835-L843, 1999.

19. Hovenberg HW, Davies JR and Carlstedt I: Different mucins are produced by the surface epithelium and the submucosa in human trachea: identification of MUC5AC as a major mucin from the goblet cells. Biochem J 318: 319-324, 1996.

20. Hovenberg HW, Davies JR, Herrmann A, Lindén CJ and Carlstedt I: MUC5AC, but not MUC2, is a prominent mucin in respiratory secretions. Glycoconj J 13: 839-847, 1996.

21. Sheehan JK, Brazeau C, Kutay S, Pigeon H, Kirkham S, Howard M and Thornton DJ: Physical characterization of the MUC5AC mucin: a highly oligomeric glycoprotein whether isolated from cell culture or in vivo from respiratory mucous secretions. Biochem J 347: 37-44, 2000.

22. Janek P, Briand P and Hartman NR: The effect of estroneprogesterone treatment on cell proliferation kinetics of hormonedependent GR mouse mammary tumors. Cancer Res 35: 3698-3704, 1975 .

23. Tsutsumida H, Swanson BJ, Singh PK, Caffrey TC, Kitajima S, Goto M, Yonezawa S and Hollingsworth MA: RNA interference suppression of MUC1 reduces the growth rate and metastatic phenotype of human pancreatic cancer cells. Clin Cancer Res 12: 2976-2987, 2006.
24. Chaturvedi P, Singh AP, Moniaux N, Senapati S, Chakraborty S, Meza JL and Batra SK: MUC4 mucin potentiates pancreatic tumor cell proliferation, survival, and invasive properties and interferes with its interaction to extracellular matrix proteins. Mol Cancer Res 5: 309-320, 2007.

25. Truant S, Bruyneel E, Gouyer V, De Wever O, Pruvot FR, Mareel $M$ and Huet G: Requirement of both mucins and proteoglycans in cell-cell dissociation and invasiveness of colon carcinoma HT-29 cells. Int J Cancer 104: 683-694, 2003.

26. Ligtenberg MJL, Buijs F, Vos HL and Hilkens J: Suppression of cellular aggregation by high levels of episialin. Cancer Res 52 : 2318-2324, 1992

27. Duan R, Remeijer L, van Dun JM, Osterhaus AD and Verjans GM: Granulocyte macrophage colony-stimulating factor expression in human herpetic stromal keratitis: implications for the role of neutrophils in HSK. Inv Ophth Vis Sci 48: 277-284, 2007.

28. Tamamori Y, Sawada T, Nishihara T, et al: Granulocyte-colony stimulating factor enhances chimeric antibody K-MAC5 dependent cytotoxicity against pancreatic cancer mediated by polymorphonuclear neutrophils. Int J Oncol 21: 649-654, 2002.

29. Lieschke GJ and Burgess AW: Drug therapy: granulocyte colony-stimulating factor and granulocyte-macrophage colonystimulating factor (1\&2). N Engl J Med 327: 28-35, 99-106, 1992.

30. Stockmeyer B, Valerius T, Repp R, et al: Preclinical studies with Fc $\gamma$ R bispecific antibodies and granulocyte colonystimulating factor-primed neutrophils as effector cells against HER-2/neu overexpressing breast cancer. Cancer Res 57: 696-701, 1997.

31. Hinoda Y, Ikematsu Y, Horinochi M, et al: Increased expression of MUC1 in advanced pancreatic cancer. J Gastroenterol 38: 1162-1166, 2003.

32. Gimmi CD, Morrison BW, Mainprice BA, et al: Brest cancer associated antigen, DF3/MUC1, induces apoptosis of activated human T cells. Nat Med 2: 1367-1370, 1996.

33. Ho JJL: Mucins in the diagnosis and therapy of pancreatic cancer. Curr Pharm Des 6: 1881-1896, 2000.

34. Inaba $\mathrm{T}$, Sano H, Kawahito Y, et al: Induction of cyclooxygenase-2 in monocyte/macrophage by mucins secreted from colon cancer cells. Proc Natl Acad Sci USA 100: 2736-2741, 2003.

35. Sugihara I, Yoshida M, Shigenobu T, et al: Different progression of tumor xenografts between mucin-producing and mucin-nonproducing mammary adenocarcinoma-bearing mice. Cancer Res 66: 6175-6182, 2006. 